\title{
Using fMRI to Assess Brain Activity in People With Down Syndrome: A Systematic Review
}

\author{
Maria Carbó-Carreté ${ }^{1,2}$, Cristina Cañete-Massé ${ }^{3,4,5}$, Maribel Peró-Cebollero ${ }^{2,3,4,5}$ and \\ Joan Guàrdia-Olmos $2,3,4,5 *$ \\ ${ }^{1}$ Department of Cognition, Development and Educational Psychology, Faculty of Psychology, University of Barcelona, \\ Barcelona, Spain, ${ }^{2}$ Institute of Neuroscience, University of Barcelona, Barcelona, Spain, ${ }^{3}$ Department of Social Psychology \\ and Quantitative Psychology, Faculty of Psychology, University of Barcelona, Barcelona, Spain, ${ }^{4}$ UB Institute of Complex \\ Systems, University of Barcelona, Barcelona, Spain, ${ }^{5}$ Quantitative Psychology Research Group (SGR 266), Generalitat de \\ Catalunya, Barcelona, Spain
}

OPEN ACCESS

Edited by:

Zhen Yuan,

University of Macau, China

Reviewed by:

Chenggang $W u$

University of Macau, China Andrés Antonio González-Garrido,

Universidad de Guadalajara, Mexico

*Correspondence:

Joan Guàrdia-Olmos jguardia@ub.edu

Specialty section

This article was submitted to Brain Imaging and Stimulation, a section of the journal

Frontiers in Human Neuroscience

Received: 17 November 2019 Accepted: 03 April 2020 Published: 27 April 2020

Citation: Carbó-Carreté M, Cañete-Massé C Peró-Cebollero $M$ and Guàrdia-Olmos J (2020) Using fMRI to Assess Brain Activity in People With

Down Syndrome: A Systematic

Review.

Front. Hum. Neurosci. 14:147 doi: 10.3389/fnhum.2020.00147
Background: In the last few years, many investigations have focused on brain activity in general and in populations with different pathologies using non-invasive techniques such as electroencefalography (EEG), positron emission tomography (PET), functional magnetic resonance imaging (fMRI) and magnetic resonance imaging (MRI). However, the use of non-invasive techniques to detect brain signals to evaluate the cognitive activity of people with Down syndrome (DS) has not been sufficiently addressed. The objective of this study is to describe the state-of-the-art in fMRI techniques for recording brain signals in people with DS.

Method: A systematic review was performed based on PRISMA recommendations; only nine papers on this topic have been published. Three independent researchers selected all relevant information from each paper. Analyses of information concordance showed a high value of agreement between researchers.

Results: Although few relevant works have been published, the use of $\mathrm{fMRI}$ in people with DS is becoming an appropriate option to study brain function in this population. Of the nine identified papers, five used task designs, and four used resting-state paradigms.

Conclusion: Thus, we emphasize the need to incorporate rigorous cognitive activity procedures in evaluations of the DS population. We suggest several factors (such as head correction movements and paired sample techniques) that must be considered when designing an $\mathrm{fMRI}$ study with a task or a resting-state paradigm in a DS population.

Keywords: down syndrome, fMRI, brain signal, brain activity, systematic review

\section{INTRODUCTION}

Analysis of the cognitive activity of people with Down Syndrome (DS) is extraordinarily relevant, and it has become a foundation for better understanding the development of neurodegenerative diseases, mainly Alzheimer's disease (AD) (Prasher et al., 1996; Lamar et al., 2011; Neale et al., 2018; Pujol et al., 2018; Musaeus et al., 2019); studying the development and morphological characteristics of the brains of individuals with DS (Baburamani et al., 2019; Lao et al., 2019; Rodrigues et al., 2019; Shiohama et al., 2019); and evaluating cognitive functioning (Virji-Babul et al., 2013). All these cited researchers used non-invasive brain registration techniques and 
extended studies that had the same objectives but used traditional paradigms (Contestabile et al., 2010; Wiseman et al., 2015). Early studies that focused on examining, for example, the relationship between $\mathrm{AD}$ and people with DS, identified certain limitations. The most notable limitation is the variability of the degree of intellectual disability of the assessed person and the problems understanding the verbal instructions of the test among people with severe or profound intellectual disability (Crayton et al., 1998; Oliver, 1999). In addition, the behavioral differences associated with dementia have an impact not only on the person with DS but also on the professionals who administer the tests, so the reliability of these responses must be examined and psychometrically guaranteed (Oliver et al., 2011). Finally, it should be noted that many psychometric scales have not been validated in populations with $\mathrm{AD}$ and $\mathrm{SD}$, but some studies have made progress in addressing these gaps (Dekker et al., 2015).

Thus, our proposal in the present work is to advance in the knowledge of all the present contributions of brain signal neuroimaging techniques in the DS population. Many studies have shown the advantages of using non-invasive brain registration techniques, as these measurements have no response bias or learning processes because when tasks are used, they have already been learned in the preregistration phases. The use of the main techniques such as EEG (electroencephalography), MRI (magnetic resonance imaging), PET (positron emission tomography), DTI (diffusion tensor imaging), and fMRI (functional magnetic resonance imaging) provide information on structural mechanisms and functional aspects, explaining the connectivity networks that can be found in healthy subjects as well as those suffering from diseases (Massoud and Gambhir, 2003; Hoehn and Aswendt, 2013; Aswendt et al., 2017).

Regarding the SD population, the systematic review presented by Neale et al. (2018) indicates that PET techniques allow the identification of amyloid accumulation prior to the onset of Alzheimer's disease (AD), while techniques based on EEG and MRI identify cognitive impairment and can be assessed as biomarkers for the detection and diagnosis of $\mathrm{AD}$ in this population.

In addition to the already cited signals, in the general population, fMRI registers have been used in the last 20 years as derivatives of the images obtained from MRI data, and certainly, the use of fMRI to assess brain activity and functioning, as well as the use of various study designs, has become common (Welvaert and Rosseel, 2014). Despite the amount of published works, we believe that it is urgent to advance this field since the data obtained from fMRI are highly valuable and their use provides unique and remarkable results in general and specific populations.

One of the most interesting advantages of studying the DS population with resting-state fMRI, as with other non-invasive techniques, is that the resting-state fMRI register does not depend on the intellectual level of the person being evaluated. In this approach, the person should only be at rest inside a resonator, without doing anything special, with his or her eyes open and without moving. In contrast, in other study designs, the fMRI signal is recorded when a specific cognitive task (e.g., language, memory, motor, among others) is being performed. Clearly, based on the uses and potential of the fMRI signal in resting-state designs (Lu et al., 2007; Biswal et al., 2010), this approach may be an interesting option because it allows the analysis of the cognitive activity of people with ID and, as in the general population, facilitates the systematic study of spontaneous fluctuations of the BOLD signal. Notably, information from studies that involve a task is derived from a direct source of activation when the participant is faced with the task set, and the resting-state data are derived from an indirect source that is not associated with the task.

It seems clear that some of the problems that occur when using fMRI signals may be aggravated for the DS population. This observation is based on the difficulties that people with DS have been reported to have when MRI data is being obtained, which suggests that with fMRI techniques, such the occurrence incidents can increase. Reviews of MRI in the DS population indicate several questions that can be applied to fMRI studies, which we can summarize in the following points. (1) It is feasible that the brain connectivity network in DS persons is more weakened than that in healthy persons of the same chronological age. We must interpret exactly what we mean by the weakening of a network, as the points of interest to determine it are diverse (e.g., density, laterality, entropy, and complexity, among other possible topics). It is also possible that the manifestation of cognitive impairment in persons with DS will, in some cases, be compensated by the intervention of other brain areas. (2) Brain volume and head size are smaller in DS persons than in healthy populations (Pinter et al., 2001; Rodrigues et al., 2019), and a smaller number of neurons and fewer synaptic extensions and altered neuronal differentiation in fetuses with DS are detected (Takashima et al., 1981; Bhattacharyya et al., 2009; Kanaumi et al., 2013). This issue has led to the use of DARTEL (Ashburner, 2007) as a template in some studies with persons with DS (Lin et al., 2014). DARTEL (Diffeomorphic Anatomical Registration through Exponentiated Lie Algebra) is a specific brain template used in the preprocessing phase or analysis of fMRI data to take into account deformations that must be parameterized by a single flow field, which is considered to be constant in time. (3) The DS population moves excessively during the registration of the signal, which leads to many experimental difficulties (Pujol et al., 2014). In this sense, Lao et al. (2019) point out an extremely important fact: the MRI signals acquired with motion correction below $1 \%$ allow the use of a general template for one of the general populations, which facilitates the study of PET signals, among others. If these assessments are documented by MRI data, it seems logical to consider them with respect to fMRI records.

Given the aforementioned points, it seems that the use of MRI data in the DS population is an interesting matter. Consequently, the question whether fMRI signals are adequate for use in this population must be addressed. The study of spontaneous fluctuations of the fMRI BOLD signal has become the goal of investigating connectivity and understanding how brain networks are organized, whether based on a stimulus response or simply at rest (Fox and Raichle, 2007; Raichle, 2009). Moreover, resting-state fMRI (rs-fMRI) has become an increasingly popular method of MRI that investigates synchronous activity across 
regions in the absence of an explicit signal correlation-based task (Corbetta, 2012; Snyder and Raichle, 2012). Data acquired by fMRI provides valuable information for explaining the determinants of network dysfunction, either with task designs to evaluate the person's different cognitive abilities (Hampson et al., 2006) or with rs-fMRI in populations that may have some diseases such as epilepsy (Centeno and Carmichael, 2014).

Therefore, based on the extensive contributions that have been shown in robust fMRI studies and the detailed review of non-invasive neuroimaging techniques in the population with DS (Baburamani et al., 2019), it is now important to establish the best experimental option to guarantee the validity and reliability of the brain signals recorded in individuals with DS. Moreover, our intention is to provide a reference point that allows us to systematically accumulate and order the available information and the findings derived from the fMRI and DS binomial (other signals such as PET or EEG are contemplated in the review of Neale et al., 2018, but this is not the case for fMRI signals.) Therefore, we want to review the works published to date in relation to fMRI data and people with DS, provide useful insights that identify the main difficulties and findings that researchers could utilize, and discuss the different ways to solve these difficulties.

\section{METHOD}

\section{Search of Published Studies}

The articles included in the present study were searched in the Web of Science (WoS), PubMed and PsycInfo databases. The following inclusion criteria were applied: the articles had to be original fMRI papers that included a sample of persons with DS and that were published from 1992 to October 17, 2019. The literature search was conducted using a Boolean algorithm with the following keywords: ("DOWN SYNDROME" OR "DOWN'S SYNDROME") in the title and ("Functional Magnetic Resonance Imag*” OR FMRI) in any part of the paper. If we added the keyword Alzheimer, no works were found in the three databases; consequently, in the present study, we worked with a combination of DS and fMRI studies. The search was performed independently by three researchers, and we obtained a $100 \%$ rate of agreement between them for the search; all papers found by the three researchers were considered in the study. Following these search criteria, we identified a total of 15 papers in WoS, 2 papers in PubMed and 19 records in PsycInfo. After duplicates were removed, a total of 9 papers were screened. None of these papers were discarded; thus, 9 articles were fully reviewed and were included in the current study (identified with an ${ }^{*}$ in the bibliography). Figure 1 presents a graph that summarizes this search process.

Three independent researchers analyzed each paper to estimate the most important information from each of the included articles. Table 1 shows the main characteristics of these articles.

As shown in Table 1, the nine works were published after 2002 , and seven studies were published in the last 9 years. Finally, the work of Seyffert et al. (2002) is a conference proceeding and therefore provides little information.

\section{RESULTS}

\section{Main Characteristics of the Studies}

Table 2 shows the main characteristics of the samples used in the nine analyzed works. As shown in the table, seven of the works are American, one is Taiwanese, and one is from the United Kingdom. The total sample sizes range between 6 and 76 participants belonging to two groups, a DS group and a control group; however, in Jacola et al. (2014) and Vega et al. (2015), three groups are used. In Jacola et al. (2014), there are two control groups, one paired by chronological age and the other by mental age; in Vega et al. (2015), there is a group of DS individuals, a group of Williams syndrome (WS) participants and a control group. In general, no characteristics of the total sample are provided; however, sample characterization is performed for each of the groups. The percentage of males, in general, is higher than $50 \%$ in both analyzed groups, even reaching $71.05 \%$ in the study by Wan et al. (2017). Notably, in the eight studies in which information on the age of the participants is available, the ages range between 5 and 47 years old. In the majority of the papers, the groups were paired by chronological age and/or sex.

Notably, in the work of Wan et al. (2017), the DS group is divided into two groups, an intervention group $(n=18)$ and a non-intervention group $(n=20)$. Because of this subdivision, the groups are not equal in terms of sex and age, so the proportion of men in the DS intervention group is $61.11 \%$, while in the DS nonintervention group, the proportion of men is $80 \%$; the average age in the DS intervention group is 14.09 years, while in the DS non-intervention group, it is 12.35 years. Finally, none of the nine studies present information on the degree of disability of the persons with DS.

\section{fMRI Description and Main Results}

As shown in Table 3, in eight of the works, the resonator used is 3 Teslas, while in the oldest work (Seyffert et al., 2002), the resonator is 1.5 Teslas. In five studies, the block design is used; therefore, the subjects must perform a task while undergoing BOLD signal acquisition. The rest of the works use the restingstate paradigm, but two of them employ unusual strategies, such as presenting visual stimuli during signal acquisition (Anderson et al., 2013, 2015). Jacola et al. (2011, 2014) and Reynolds Losin et al. (2009) use semantic listening type tasks, whereas in the Wan et al. (2017) study, visual perception tasks are used. The amount of time that persons are in the resonator performing the task is generally short, ranging from $5 \mathrm{~min} 30 \mathrm{~s}$ in the shortest case to $50 \mathrm{~min}$ in the longest case. In general, the objective of the analyzed works is to determine if there is a differentiated activation pattern between the DS group and the analyzed control group. Clearly, the papers estimating functional connectivity networks try to show the difference between groups in relation to network patterns and characteristics.

A summary of the results found in the different works is also provided in Table 3. The task description of these works is noteworthy since none of them mention the previous learning periods, and the level of difficulty of the task is not indicated in all of them. In fact, in older works using a resting-state approach, visualization is used to avoid excessive movement. The extent to which these sequences would be considered 


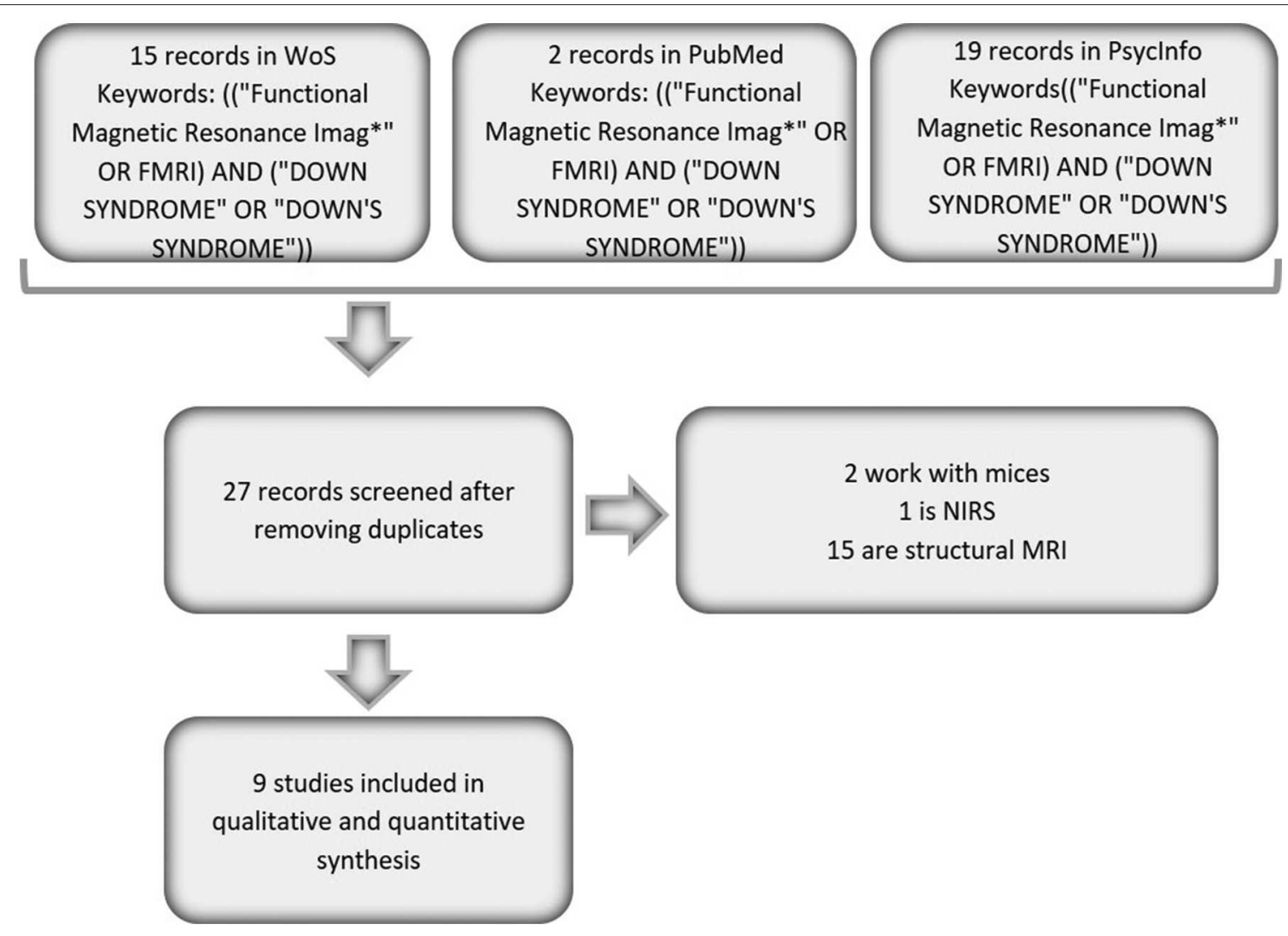

FIGURE 1 | Flow chart of the analyzed papers.

resting is debatable, since these types of records, as we have reiterated, occur in the absence of any external stimulation. An examination of the concrete results of each of the papers indicates some common characteristics, even though the papers are not strictly comparable. In general, differential activation patterns are seen in the DS samples compared to the control groups. This pattern is not regular, and statistically significant differences are observed in unilateral comparisons in both the Down $>$ Control and Control $>$ Down assessments. Most likely, depending on the characteristics of the tasks, distinct and small extrapolated activation patterns were obtained. For example, Seyffert et al. (2002) found greater activation in the DS sample than in the control group in a task to silently name pictures of common objects. On the other hand, Reynolds Losin et al. (2009), in a passive story-listening task (Blocks: Forward and Backward and Rest), found similar effects in some comparisons. For example, in the Forward $>$ Backward task, statistically significant effects were found in the unilateral comparisons of the Control > DS groups. However, in other tasks (Forward $>$ Rest), the statistically significant difference was in the opposite direction DS $>$ Control. The works of Jacola et al. $(2011,2014)$ were especially consistent since they only found statistically significant effects in the Control $>$ DS comparisons in all the areas studied. Inconsistent and irregular activation patterns, as we mentioned, can be seen in the remaining works (Vega et al., 2015; Wan et al., 2017; Wilson et al., 2019). The two works carried out with a resting-state approach (Anderson et al., 2015) showed less activation in the functional connectivity networks presented in the DS samples. However, these results are not comparable since both studies used resting-state techniques with the presentation of visual stimuli.

\section{DISCUSSION}

We should wait a few more years for more studies using fMRI techniques to be published and to include various specific populations. Even so, it is quite pertinent to promote the use of these techniques, which deserve special attention in the DS population for their singularities in relation to their cognitive functioning and intellectual level. Generally, the increase in the use of neuroimaging techniques has led to the appearance of many underpowered studies with small sample sizes, which leads to many missed results (Button et al., 2013). This leads us to continue to explore the different findings.

Presently, it is optimistic to talk about generalized conclusions given the small amount of evidence available (eight or nine papers depending on the consideration of Seyffert et al., 2002). In any case, we aim to provide useful reference points to support future work that utilizes fMRI techniques with the DS population. 
TABLE 1 | Main characteristics of the analyzed papers.

\begin{tabular}{|c|c|c|c|}
\hline Title & Authors & Year & Journal \\
\hline Abnormal brain synchrony in Down Syndrome. & $\begin{array}{l}\text { Andesrson J. S., Nielsen J. A., Ferguson M. A., } \\
\text { Burback M. C., Cox E. T., Dai L. et al. }\end{array}$ & 2013 & Neurolmage \\
\hline $\begin{array}{l}\text { Violence: heightened brain attentional network response is selectively } \\
\text { muted in Down syndrome. }\end{array}$ & $\begin{array}{l}\text { Anderson J. S., Treiman S. M., Ferguson M. A., } \\
\text { Nielsen J. A., Edgin J. O., Dai L. et al. }\end{array}$ & 2015 & $\begin{array}{l}\text { Journal of Neurodevelopmental } \\
\text { Disorders }\end{array}$ \\
\hline $\begin{array}{l}\text { Functional magnetic resonance imaging of cognitive processing in } \\
\text { young adults with Down syndrome. }\end{array}$ & $\begin{array}{l}\text { Jacola L. M., Byars A. W., Chalfonte-Evans M., } \\
\text { Schmithrst V. J., Hickey F., Patterson, B. et al. }\end{array}$ & 2011 & $\begin{array}{l}\text { American Journal on Intellectual } \\
\text { and Developmental Disabilities }\end{array}$ \\
\hline $\begin{array}{l}\text { Functional magnetic resonance imaging of story listening in } \\
\text { adolescents and young adults with Down syndrome: evidence for } \\
\text { atypical neurodevelopment. }\end{array}$ & $\begin{array}{l}\text { Jacola L. M., Byars A. W., Hickey F., Vannest J., } \\
\text { Holland S. K. and Schapiro M. B. }\end{array}$ & 2014 & $\begin{array}{l}\text { Journal of Intellectual Disability } \\
\text { Research }\end{array}$ \\
\hline $\begin{array}{l}\text { Abnormal fMRI activation pattern during story listening in individuals } \\
\text { with Down syndrome. }\end{array}$ & $\begin{array}{l}\text { Reynolds Losin E. A., Rivera S. M., O'Hare E. D., } \\
\text { Sowell E. R. and Pinter J. D. }\end{array}$ & 2009 & $\begin{array}{l}\text { American Journal on Intellectual } \\
\text { and Developmental Disabilities }\end{array}$ \\
\hline $\begin{array}{l}\text { Functional magnetic resonance imaging shows aberrant language } \\
\text { lateralization in Down syndrome. }\end{array}$ & Seyffert M., Field K. and Pinter J. & 2002 & Annals of Neurology \\
\hline $\begin{array}{l}\text { Resting-state functional connectivity in individuals with Down syndrome } \\
\text { and Williams syndrome compared with typically developing controls. }\end{array}$ & $\begin{array}{l}\text { Vega J. N., Hohman T. J., Pryweller J. R., Dykens } \\
\text { E. M., and Thornton-Wells T. A. }\end{array}$ & 2015 & Brain Connectivity \\
\hline $\begin{array}{l}\text { The effectiveness of the computerized visual perceptual training } \\
\text { program on individuals with Down syndrome: An fMRI study. }\end{array}$ & $\begin{array}{l}\text { Wan Y.T., Chiang C. S., Chen S. C. and Wuang Y. } \\
\text { P. }\end{array}$ & 2017 & $\begin{array}{l}\text { Research in Developmental } \\
\text { Disabilities }\end{array}$ \\
\hline $\begin{array}{l}\text { Differential effects of Down's syndrome and Alzheimer's } \\
\text { neuropathology on default mode connectivity. }\end{array}$ & $\begin{array}{l}\text { Wilson L. R., Vatansever D., Annus T., Williams G. } \\
\text { B., Hong Y. T., Fryer T. D. et al. }\end{array}$ & 2019 & Human Brain Mapping \\
\hline
\end{tabular}

First, there is evidence of functional and structural differences between populations. Lower brain volume and lower activity (activation) recorded by fMRI appear to be typical in the DS population. It is evident that the morphological differences in the brain of a person with DS brain fuel the discussion about the normalized atlases, which were also revealed in the MRI studies of persons with DS. For instance, Pujol et al. (2018) used SPM voxel-based morphometry (VBM) with DARTEL algorithms in the image preprocessing phase.

Second, related to the last point, in fMRI sessions, we have already reiterated that the recording problems stem primarily from the movement of the person within the resonator. This problem is common in children and in populations with pathologies that compromise motor control (Aranyi et al., 2017). This tendency is observed among DS persons. As a consequence, some records have to be eliminated, or statistical routines are required to correct broadband movement in the preprocessing phase. It is a source of noise to consider in this type of work. Except for the work of Wilson et al. (2019), this issue is not mentioned in the rest of the works. In this regard, the proposals of Ciric et al. (2017) should be taken into account in all fMRI studies and with more intensity in high-movement populations such as persons with DS. Even if their suggestions do not provide specific corrections for DS samples, they have been shown to be effective in reducing the perverse effects of excess movement. The work of Wilson et al. (2019) may be a reference for the appropriate application of these corrections.

Third, in the same manner, the sample sizes are small. This finding is not new; it is a recurring theme in many works, not necessarily just DS studies. Therefore, this factor is not a distinctive aspect of these samples. The difficulties of sampling are well-known but are not different from many other proposals for fMRI. From a classical perspective, the expected minor sampling error in this work would not be inferior to 0.1124 assuming a CI of $95 \%$ and a theoretical parameter $\pi=0.5$.

From a methodological point of view, and as the fourth issue, we can identify certain doubts in the configuration of control groups of healthy people in the employed designs. It is important to note that the mental development of subjects with intellectual disability is not the same as that of healthy subjects of equal chronological age but should not differ significantly when matched for their mental age (Carducci et al., 2013). The focus is on the selection of an appropriate control group, and the options are matching by chronological age or by mental age. In the first case, we consider a non-specific maturation process, and in the second, we address cognitive skills, which are also developmental but focus fundamentally on performance. Notably, in the case of studies that involve tasks, it would be appropriate to promote having control groups matched by mental age, as it is a question of comparing cognitive performance and brain signals (Jacola et al., 2014). Only one study analyzed described two paired control groups, one by chronological age and another by mental age, because basal functioning was being evaluated rather than factors associated with an explicit cognitive task or component. In this respect, there is one aspect that is of little or no consideration. If a control group is generated from the estimation of mental age matching, strict matching must be performed (consisting with having an exact mirror in the control group of each case in the experimental group). If a poorly matched group is used due to sampling difficulties, the control is usually verified by comparing the means of the mental age between the two groups; relevantly, IQ ranges differ greatly between individuals with and without DS. In people without ID, the IQ ranges between 85 and 115 in almost $66 \%$ of people (according to the normal curve properties); the population of ID individuals is usually distributed into those that exhibit slight or medium delays, that 
TABLE 2 | Sample description of the analyzed papers.

\begin{tabular}{|c|c|c|c|c|c|c|c|c|c|}
\hline Paper & Sample Country & $n$ & $\%$ men & Groups & n by group & $\%$ men by group & $\begin{array}{c}\text { Age mean by } \\
\text { group }\end{array}$ & Sampling & Matching \\
\hline Anderson et al. (2013) & USA & 29 & 58.62 & 2 & $\begin{array}{l}\text { DS: } 15 \\
\text { C: } 14\end{array}$ & $\begin{array}{l}\text { DS: } 60.00 \\
\text { C: } 57.14\end{array}$ & $\begin{array}{c}\text { DS: } 20.20 \\
\text { C: } 23.70\end{array}$ & Accidental & $\begin{array}{l}\text { Chronological age } \\
\text { and sex }\end{array}$ \\
\hline Anderson et al. (2015) & USA & 29 & 58.62 & 2 & $\begin{array}{c}\text { DS: } 15 \\
\text { C: } 14\end{array}$ & $\begin{array}{l}\text { DS: } 60.00 \\
\text { C: } 57.14\end{array}$ & $\begin{array}{l}\text { DS: } 20.20 \\
\text { C: } 23.70\end{array}$ & Accidental & $\begin{array}{l}\text { Chronological age } \\
\text { and sex }\end{array}$ \\
\hline Jacola et al. (2011) & USA & 25 & 60.00 & 2 & $\begin{array}{l}\text { DS: } 13 \\
\text { C: } 12\end{array}$ & $\begin{array}{l}\text { DS: } 61.54 \\
\text { C: } 58.33\end{array}$ & $\begin{array}{l}\text { DS: } 18.30 \\
\text { C: } 19.00\end{array}$ & Accidental & $\begin{array}{l}\text { Chronological age } \\
\text { and sex }\end{array}$ \\
\hline Jacola et al. (2014) & USA & 36 & 47.22 & 3 & $\begin{array}{l}\text { DS: } 11 \\
\text { C_CA: } 13 \\
\text { C_MA: } 12\end{array}$ & $\begin{array}{c}\text { DS: } 54.54 \\
\text { C_CA: } 53.85 \\
\text { C_MA: } 33.33\end{array}$ & $\begin{array}{c}\text { DS: } 18.3 \\
\text { C_CA: } 18.3 \\
\text { C_MA: } 5.4\end{array}$ & Accidental & $\begin{array}{l}\text { C_CA: } \\
\text { chronological age } \\
\text { C_MA: mental age }\end{array}$ \\
\hline Reynolds Losin et al. (2009) & USA & 18 & 50.00 & 2 & $\begin{array}{c}\text { DS: } 9 \\
\text { C:9 }\end{array}$ & $\begin{array}{c}\text { DS: } 44.44 \\
\text { C: } 55.56\end{array}$ & $\begin{array}{l}\text { DS: } 16.5 \\
\text { C: } 17.8\end{array}$ & Accidental & Chronological age \\
\hline Seyffert et al. (2002) & USA & 6 & & 2 & $\begin{array}{c}\text { DS: } 3 \\
\text { C: } 3\end{array}$ & & & Accidental & \\
\hline Vega et al. (2015) & USA & 68 & 60.29 & 3 & $\begin{array}{l}\text { DS: } 10 \\
\text { WS: } 18 \\
\text { C: } 40\end{array}$ & $\begin{array}{c}\text { DS: } 40 \\
\text { WS: } 72.22 \\
\text { C: } 60.00\end{array}$ & $\begin{array}{c}\text { DS: } 38.98 \\
\text { WS: } 25.89 \\
\text { C: } 46.95\end{array}$ & Accidental & \\
\hline Wan et al. (2017) & Taiwan & 76 & 71.05 & 2 & $\begin{array}{c}\text { DS: } 38 \\
\text { C: } 38 \\
\text { The DS group was } \\
\text { divided in two } \\
\text { samples: } \\
\text { intervention }(n=18) \\
\text { and control }(n=20)\end{array}$ & $\begin{array}{l}\text { DS: } 71.05 \\
\text { C: } 71.05\end{array}$ & $\begin{array}{l}\text { DS: } 13.17 \\
\text { C: } 13.07\end{array}$ & Accidental & $\begin{array}{l}\text { Chronological age } \\
\text { and sex }\end{array}$ \\
\hline Wilson et al. (2019) & United Kingdom & 54 & 50.00 & 2 & $\begin{array}{c}\text { DS: } 34 \\
\text { C:20 }\end{array}$ & $\begin{array}{l}\text { DS: } 55.00 \\
\text { C: } 47.00\end{array}$ & $\begin{array}{c}\text { DS: } 43.5 \\
\text { C_CA: } 43.5\end{array}$ & Accidental & Chronological age \\
\hline
\end{tabular}

DS, Down syndrome group; C, control group; C_CA, control group matched by chronological age; C_MA, control group matched by mental age; WS, Williams syndrome group.

is, those with IQs up to 70 in most cases. In addition, IQs are stable in the adult population but much less stable in the child population; thus, comparison with this type of matching can be misleading (Amador and Forns, 2019). No matching analyses have been reported with empirical evidence of homoscedasticity between groups paired with lax criteria.

In this sense, as a fifth point, the works analyzed here show comparisons between groups of statistically significant activations or networks obtained from the fMRI records during a semantic recognition task, passive listening, non-verbal denomination of objects, a visual perception task or a resting state (see Table 3). In all cases, activation differences are reported in certain areas of the brain with intergroup comparison. The fact that the visual areas are activated less in the DS sample than in the control group or that there are differences in other areas due to visual stimuli does not indicate specific properties of the DS population. The same consideration must be addressed in network differences. In fact, in general, all studies agree in reporting a lower activation intensity in DS groups than control groups, and the study by Wan et al. (2017) presents an interesting intragroup comparison that does describe intrinsic properties of the DS population. However, the lower activation level obtained in the DS group than in the controls confirmed the effects of brain impoverishment described above. We can make many comparisons with many different stimuli; however, these comparisons will not directly lead us to discovering unique brain behavior properties of the DS population. In fact, an activation increment is described in some connections, and a decrement is noted in others in the comparisons between networks in DS samples and the paired control groups. However, these differences do not allow the establishment of a stable and regular pattern typical of DS people.

These findings lead us to another crucial point. The use of tasks that previously published papers have used are tasks adapted to the peculiarities of the target population. Comparison with a healthy control group of similar chronological age does not in any way support characteristic outcomes among the DS population. The comparison between DS and non-DS groups does not provide relevant evidence regarding individuals with DS. It seems more methodologically reasonable to make intragroup comparisons than intergroup comparisons and, when feasible, use internal classification criteria for the identification of subgroups. For instance, to compare older and younger individuals, those with higher cognitive competence and those with lower competence, those with a higher cognitive reserve level and those with a lower level and so on within the DS population, any other criteria that allows characterization of cognitive competence by signals and study of its distribution should be utilized.

Intragroup comparisons present other types of statistical issues that extend beyond the objective of this paper but are reasonably resolved and can be used in DS studies. We can observe examples of these issues in AD or Parkinson's populations or in populations with other pathologies with 
TABLE 3 | Principal characteristics of the fMRI design and principal results obtained.

Teslas Design

Task

Main aim

Anderson et al. (2013) $\quad$ 3T Resting-state imaging with visualizing cartoons
Paradigm that required participants to
make a decision based on semantic

information derived from visually presented stimuli

Language processing: a passive story 5'30" listening paradigm

Passive story-listening task (Blocks: forward, backward and rest)

Silent naming of pictures of common
Compare fMRI scans of 15 individuals with Down syndrome to 14 typically developing control subjects while they viewed cartoon video clips response to stylized violence stimuli in syndrome and in typically developing individuals to determine whether regional brain activation patterns could be characterized, as well as whether atypical Measurements of subject motion were significantly higher in Down syndrome subjects than in controls. Down syndrome subjects showed higher levels of synchrony between distributed brain networks as well as between the vast majority of gray matter regions. Down syndrome subjects exhibited weaker correlations only for a relatively small subset of the most correlated regions, whether negatively or positively related. Regardless of the distance separating the regions, pairs of regions that showed anticorrelation in a large control sample showed increased correlation (reduced anticorrelation) in Down syndrome.

In typically developing individuals, the brain's dorsal attention network was most active during violent scenes in the cartoons; this was significantly and specifically reduced in Down syndrome participants. Individuals with Down syndrome exhibited significantly reduced activation in the primary sensory cortices, and such neural activation might be present that could perceptual impairments may constrain their ability to provide clues to a basis for the deficits seen respond to more complex social cues such as violence. in Down syndrome

Understand the relationship between

A significant difference was present in task performance cognitive processing and brain activation in between the mean of DS and control individuals; the individuals with Down syndrome on tasks mean of DS individuals was inferior to that of controls. In that measured aspects of both verbal and relation to fMRI, controls had 13 areas activated, visual-spatial abilities relation to fMRI, controls had 13 areas

Explore neural activation during language Random effects group analyses documented a reduced processing in participants with DS compared activation magnitude in the DS cohort than in both with typically developing groups matched for control groups. The pattern of activation within the DS chronological and mental age cohort additionally included significantly greate activation in the midline frontal regions (BA 9 and 10) and cingulate gyri (BA 23, 24, 30 and 32).
Investigate whether individuals with DS exhibit aberrant language-related activatio patterns compared to an approximately age-matched typically developing contro group during an easily performed passive story-listening task

Unspecific objective related to language deficits in Down syndrome

Control > DS: Forward > Backward-Right middle temporal gyrus.

DS > Control: Forward > Rest-Right precuneus Backward $>$ Rest-Right precuneus. objects presented through fiber-optic goggles. As a control condition,

subjects viewed pixilated images of the same objects with instructions to look without attempting to name them 
Vega et al. (2015) 3T Resting state
Two types of visual perceptual tasks: two-choice revised version of Hooper Visual Organization Test (T-HVOT) and Full Picture Matching Test (FPMT)
First aim: confirm previous findings of

increased between-network connectivity in DS individuals compared with TD controls and determine whether such differences are specific to DS or are also observed in anoth developmental disability disorders, such as WS.

Characterize how the within-network connectivity profiles of DS and WS could be compared with each other and with TD participants. Together, these aims are intended to support the replication of previous work while providing new insights into resting-state brain function across two different neurodevelopmental disorders.

1) Develop and implement a one-year computerized visual perceptual training (CVPT) program for DS, (2) use a

standardized visual perception assessment to evaluate the effectiveness of the CVPT program, and (3) examine the changes of cortical activation patterns of DS individuals after one-year of CVPT intervention by utilizing functional magnetic resonance imaging (fMRI)

(a) Determine the potential functional
The results showed that alterations of between-network connectivity, particularly in the DMN, are a characteristic of a number of neurodevelopmental disorders involving intellectual disability, including DS and WS. Perhaps within-network connectivity is a feature that shows more variable patterns across different neurodevelopmental disorders. with Down syndrome; (b) examine the profile of DMN connectivity; almost no anti-correlation with other cortical regions w age, $I Q$ and perfomance on memory and executive function tasks in people with Down syndrome; and (c) investigate differences in DMN connectivity in people with Down syndrome with and without fibrillar $A \beta$ accumulation, indicative of Alzheimer's disease neuropathology
The results showed that the DS intervention group had significant improvements in TVPS-3 after the

intervention. The fMRI results indicated more activation in the superior and inferior parietal lobes (spatial

manipulation), as well as the precentral gyrus and dorsal premotor cortex (motor imagery) in the DS intervention group. In the T-HVOT vs. FPMT comparison, TD individuals showed highly significant bilateral activations in the middle occipital gyrus, middle temporal gyrus, middle frontal gyrus, and inferior frontal gyrus. of Alzheimer's disease neuropathology.

\section{of}

(1)


characteristic intellectual deficits, such as Williams syndrome, Fragile FXS syndrome, Rett syndrome and Turner's syndrome (Beaton et al., 2010; Thornton-Wells et al., 2010; Chai et al., 2012; Stevenson et al., 2012; Venuti et al., 2012; Klabunde et al., 2015; Reynolds et al., 2015).

While previously mentioned, it is surprising that there are scarce resting-state fMRI records. Several studies have shown the relationship between the default mode network (DMN) and healthy aging processes; thus, the estimation of this type of wellknown network should also be a good vehicle for the study of brain functioning through fMRI in the DS population (FarràsPermanyer et al., 2019), which leads to a final point that we must propose. The study of brain signals in pathologies with cognitive deficits is common; however, it is not commonly used to evaluate people with DS, and the lack of studies with this approach cannot be explained by the difficulties of sampling or the workload of registration alone. The sampling difficulties and workload are the same those for other pathologies, but there are more published studies on people with other diagnosis than on people with DS.

The issue is that if fMRI (or other types of signals) data are used as indicators of cognitive status, they are likely to be perceived as being unnecessary in these (and other) cases. However, this is not the essential question. The conception of the brain signal cannot currently be conceived as the study of a specific activity associated with a stimulus. The nature of brain function requires a much broader consideration than designs associated with tasks in which the statistical detection of increased activation in a specific area of the brain through intergroup or intragroup comparisons is a priority. Our proposal is based on the need to study functional and effective connectivity networks for the whole brain at rest and in series that are not less than $5 \mathrm{~min}$, according to the recommendations of Cole et al. (2010) and Van Dijk et al. (2010). This recommendation provides a measure of brain functionality (i.e., connectivity networks) in a short and feasible time and with no bias.

\section{REFERENCES}

Amador, J. A., and Forns, M. (2019). Escala de Inteligencia de Wechsler Para Niños. Quinta Edición: WISC-V. Available online at: diposit.ub.edu/dspace/handle/ 2445/127676 (accessed May 15, 2019).

*Anderson, J. S., Nielsen, J. A., Ferguson, M. A., Burback, M. C., Cox, E. T., Dai, L., et al. (2013). Abnormal brain synchrony in down syndrome. Neuroimage Clin. 2, 703-715. doi: 10.1016/j.nicl.2013.05.006

*Anderson, J. S., Treiman, S. M., Ferguson, M. A., Nielsen, J. A., Edgin, J. O., Dai, L., et al. (2015). Violence: heightened brain attentional network response is selectively muted in down syndrome. J. Neurodev. Disord. 7:15. doi: 10.1186/s11689-015-9112-y

Aranyi, C., Opposits, G., Nagy, M., Berényi, E., Vér, C., Csiba, L., et al. (2017). Population-level correction of systematic motion artifacts in fMRI in patients with ischemic stroke. J. Neuroimaging 27, 397-408. doi: 10.1111/jon.12408

Ashburner, J. (2007). A fast diffeomorphic image registration algorithm. Neuroimage 38, 95-113. doi: 10.1016/j.neuroimage.2007.07.007

Aswendt, M., Schwarz, M., Abdelmoula, W. M., Dijkstra, J., and Dedeurwaerdere, S. (2017). Whole-brain microscopy meets in vivo neuroimaging:

\section{CONCLUSIONS}

To conclude, we want to summarize the notable points discuss above. First, it is feasible to use fMRI signals in a population with DS, as long as measures are provided with the utmost rigor. Second, it seems preferable to use the resting-state paradigm in this population. Third, it would be beneficial to invest time and effort in studying how the brain signal in the population with DS could be used as a biomarker of cognitive activities. Fourth, this type of data must be exhaustively analyzed to estimate classification and discrimination functions between differential groups within the same population of DS. Techniques such as linear or non-linear discriminant analysis and latent profile analysis, among others, may be especially relevant. Finally, we must remember that the techniques related to movement reduction can help improve recruitment and sampling, especially reducing experimental mortality due to register errors. All these questions are very limited thus far with respect to the effort to meta-analyze data from fMRI signal studies in populations with DS.

\section{DATA AVAILABILITY STATEMENT}

The datasets generated for this study are available on request to the corresponding author.

\section{AUTHOR CONTRIBUTIONS}

All authors have contributed to all parts of the paper: in the selection of works, the extraction of information and analysis and the writing and review of the work.

\section{FUNDING}

Project number: PGC2018-095829-B-I00 of Ministerio de Ciencia, Innovación y Universidades. Agencia Estatal de Investigación.

techniques, benefits, and limitations. Mol. Imaging Biol. 19, 1-9. doi: 10.1007/s11307-016-0988-Z

Baburamani, A. A., Patkee, P. A., Arichi, T., and Rutherford, M. A. (2019). New approaches to studying early brain development in down syndrome. Dev. Med. Child Neurol. 61, 867-879. doi: 10.1111/dmcn.14260

Beaton, E. A., Stoddard, J., Lai, S., Lackey, J., Shi, J., Ross, J. L., et al. (2010). Atypical functional brain activation during a multiple object tracking task in girls with Turner syndrome: neurocorrelates of reduced spatiotemporal resolution. Am. J. Intellect. Dev. Disabil. 115, 140-156. doi: 10.1352/1944-7558-115.2.140

Bhattacharyya, A., McMillan, E., Chen, S. I., Wallace, K., and Svendsen, C. N. (2009). A critical period in cortical interneuron neurogenesis in down syndrome revealed by human neural progenitor cells. Dev. Neurosci. 31, 497-510. doi: 10.1159/000236899

Biswal, B. B., Mennes, M., Zuo, X. N., Gohel, S., Kelly, C., Smith, S. M., et al. (2010). Toward discovery science of human brain function. Proc. Natl. Acad. Sci. U.S.A. 107, 4734-4739. doi: 10.1073/pnas.0911855107

Button, K. S., Ioannidis, J. P., Mokrysz, C., Nosek, B. A., Flint, J., Robinson, E. S., et al. (2013). Power failure: why small sample size undermines the reliability of neuroscience. Nat. Rev. Neurosci. 14:365. doi: 10.1038/nrn3475 
Carducci, F., Onorati, P., Condoluci, C., Di Gennaro, G., Quarato, P. P., Pierallini, A., et al. (2013). Whole-brain voxel-based morphometry study of children and adolescents with down syndrome. Funct. Neurol. 28:19. doi: 10.11138/FNeur/2013.28.1.019

Centeno, M., and Carmichael, D. W. (2014). Network connectivity in epilepsy: resting state fMRI and EEG-fMRI contributions. Front. Neurol. 5:93. doi: 10.3389/fneur.2014.00093

Chai, X. J., Castañón, A. N., Ongür, D., and Whitfield-Gabrieli, S. (2012). Anticorrelations in resting state networks without global signal regression. Neuroimage 59, 1420-1428. doi: 10.1016/j.neuroimage.2011.08.048

Ciric, R., Wolf, D. H., Power, J. D., Roalf, D. R., Baum, G. L., Ruparel, K., et al. (2017). Benchmarking of participant-level confound regression strategies for the control of motion artifact in studies of functional connectivity. Neuroimage 154, 174-187. doi: 10.1016/j.neuroimage.2017.03.020

Cole, D. M., Smith, S. M., and Beckmann, C. F. (2010). Advances and pitfalls in the analysis and interpretation of resting-state FMRI data. Front. Syst. Neurosci. 4:8. doi: 10.3389/fnsys.2010.00008

Contestabile, A., Benfenati, F., and Gasparini, L. (2010). Communication breaksdown: from neurodevelopment defects to cognitive disabilities in down syndrome. Prog. Neurobiol. 91, 1-22. doi: 10.1016/j.pneurobio.2010.01.003

Corbetta, M. (2012). Functional connectivity and neurological recovery. Dev. Psychobiol. 54, 239-253. doi: 10.1002/dev.20507

Crayton, L., Oliver, C., Holland, A., Bradbury, J., and Hall, S. (1998). The neuropsychological assessment of age related cognitive deficits in adults with down's syndrome. J. Appl. Res. Intellect. Disabil. 11, 255-272. doi: 10.1111/j.1468-3148.1998.tb00066.x

Dekker, A. D., Strydom, A., Coppus, A. M., Nizetic, D., Vermeiren, Y., Naudé P. J., et al. (2015). Behavioural and psychological symptoms of dementia in down syndrome: early indicators of clinical Alzheimer's disease? Cortex 73, 36-61. doi: 10.1016/j.cortex.2015.07.032

Farràs-Permanyer, L., Mancho-Fora, N., Montalà-Flaquer, M., Bartrés-Faz, D., Vaqué-Alcázar, L., Peró-Cebollero, M., et al. (2019). Age-related decrease in resting-state functional connectivity in older groups. Neural Regen. Res. 14, 1544-1555. doi: 10.4103/1673-5374.255976

Fox, M. D., and Raichle, M. E. (2007). Spontaneous fluctuations in brain activity observed with functional magnetic resonance imaging. Nat. Rev. Neurosci. 8, 700-711. doi: 10.1038/nrn2201

Hampson, M., Driesen, N. R., Skudlarski, P., Gore, J. C., and Constable, R. T. (2006). Brain connectivity related to working memory performance. J. Neurosci. 26, 13338-13343. doi: 10.1523/JNEUROSCI.3408-06.2006

Hoehn, M., and Aswendt, M. (2013). Structure-function relationship of cerebral networks in experimental neuroscience: contribution of magnetic resonance imaging. Exp. Neurol. 242, 65-73. doi: 10.1016/j.expneurol.2012.04.014

*Jacola, L. M., Byars, A. W., Chalfonte-Evans, M., Schmithorst, V. J., Hickey, F., Patterson, B., et al. (2011). Functional magnetic resonance imaging of cognitive processing in young adults with Down syndrome. Am. J. Intellect. Dev. Disabil. 116, 344-359. doi: 10.1352/1944-7558-116.5.344

*Jacola, L. M., Byars, A. W., Hickey, F., Vannest, J., Holland, S. K., and Schapiro, M. B. (2014). Functional magnetic resonance imaging of story listening in adolescents and young adults with Down syndrome: evidence for atypical neurodevelopment. J. Intellect. Disabil. 58, 892-902. doi: 10.1111/jir.12089

Kanaumi, T., Milenkovic, I., Adle-Biassette, H., Aronica, E., and Kovacs, G. G. (2013). Non-neuronal cell responses differ between normal and down syndrome developing brains. Int. J. Dev. Neurosci. 31, 796-803. doi: 10.1016/j.ijdevneu.2013.09.011

Klabunde, M., Saggar, M., Hustyi, K. M., Kelley, R. G., Reiss, A. L., and Hall, S. S. (2015). Examining the neural correlates of emergent equivalence relations in Fragile X syndrome. Psychiatry Res. 233, 373-379. doi: 10.1016/j.pscychresns.2015.06.009

Lamar, M., Foy, C. M., Beacher, F., Daly, E., Poppe, M., Archer, N., et al. (2011). Down syndrome with and without dementia: an in vivo proton magnetic resonance spectroscopy study with implications for Alzheimer's disease. Neuroimage 5, 63-68. doi: 10.1016/j.neuroimage.2011.03.073

Lao, P. J., Handen, B. L., Betthauser, T. J., Cody, K. A., Cohen, A. D., Tudorascu, D. L., et al. (2019). Imaging neurodegeneration in down syndrome: brain templates for amyloid burden and tissue segmentation. Brain Imaging Behav. 13, 345-353. doi: 10.1007/s11682-018-9888-y
Lin, L., Jin, C., and Wu, S. (2014). "Spatial covariance analysis for non-demented adults with down syndrome," in Proceeding of the 11th World Congress on Intelligent Control and Automation. (Shenyang: IEEE), 5108-5111).

Lu, H., Zuo, Y., Gu, H., Waltz, J. A., Zhan, W., Scholl, C. A., et al. (2007). Synchronized delta oscillations correlate with the resting-state functional MRI signal. Proc. Natl. Acad. Sci. U.S.A. 104, 18265-18269. doi: 10.1073/pnas.0705791104

Massoud, T. F., and Gambhir, S. S. (2003). Molecular imaging in living subjects: seeing fundamental biological processes in a new light. Genes Dev. 17, 545-580. doi: $10.1101 / \operatorname{gad} .1047403$

Musaeus, C. S., Salem, L. C., Sabers, A., Kjaer, T. W., and Waldemar, G. (2019). Associations between electroencephalography power and Alzheimer's disease in persons with down syndrome. J. Intellect. Disabil. Res. 68, 947-960. doi: $10.1111 /$ jir. 12627

Neale, N., Padilla, C., Fonseca, L. M., Holland, T., and Zaman, S. (2018). Neuroimaging and other modalities to assess Alzheimer's disease in down syndrome. NeuroImage Clin. 17, 263-271. doi: 10.1016/j.nicl.2017.10.022

Oliver, C. (1999). "Perspectives on assessment and evaluation," in Dementia. Ageing and Intellectual Disabilities: A Handbook, eds M. P. Janicki, and A. J. Dalton (Philadelphia: Bruner Mazel), 123-140.

Oliver, C., Kalsy, S., McQuillan, S., and Hall, S. (2011). Behavioural excesses and deficits associated with dementia in adults who have down syndrome. J. Appl. Res. Intellect. Disabil. 24, 208-216. doi: 10.1111/j.1468-3148.2010.00604.x

Pinter, J. D., Eliez, S., Schmitt, J. E., Capone, G. T., and Reiss, A. L. (2001). Neuroanatomy of down's syndrome: a high-resolution MRI study. Am. J. Psychiatry 158, 1659-1665. doi: 10.1176/appi.ajp.158.10.1659

Prasher, V. P., Barber, P. C., West, R., and Glenholmes, P. (1996). The role of magnetic resonance imaging in the diagnosis of Alzheimer disease in adults with down syndrome. Arch. Neurol. 53, 1310-1313. doi: 10.1001/archneur.1996.00550120122028

Pujol, J., Fenoll, R., Ribas-Vidal, N., Martínez-Vilavella, G., Blanco-Hinojo, L., García-Alba, J., et al. (2018). A longitudinal study of brain anatomy changes preceding dementia in down syndrome. NeuroImage Clin. 18, 160-166. doi: 10.1016/j.nicl.2018.01.024

Pujol, J., Macià D., Blanco-Hinojo, L., Martínez-Vilavella, G., Sunyer, J., de la Torre, R., et al. (2014). Does motion-related brain functional connectivity reflect both artifacts and genuine neural activity? NeuroImage 101, 87-95. doi: 10.1016/j.neuroimage.2014.06.065

Raichle, M. E. (2009). A paradigm shift in functional brain imaging. J. Neurosci. 29, 12729-12734. doi: 10.1523/JNEUROSCI.4366-09.2009

*Reynolds Losin, E. A., Rivera, S. M., O’Hare, E. D., Sowell, E. R., and Pinter, J. D. (2009). Abnormal fMRI activation pattern during story listening in individuals with down syndrome. Am. J. Intellect. Dev. Disabil. 114, 369-380. doi: 10.1352/1944-7558-114.5.369

Reynolds, J. E., Licari, M. K., Billington, J., Chen, Y., Aziz-Zadeh, L., Werner, J., et al. (2015). Mirror neuron activation in children with developmental coordination disorder: a functional MRI study. Int. J. Dev. Neurosci. 47, 309-319. doi: 10.1016/j.ijdevneu.2015.10.003

Rodrigues, M., Nunes, J., Figueiredo, S., de Campos, A. M., and Geraldo, A. F. (2019). Neuroimaging assessment in down syndrome: a pictorial review. Insights Imaging 10:52. doi: 10.1186/s13244-0190729-3

*Seyffert, M., Field, K., and Pinter, J. (2002). Functional magnetic resonance imaging shows aberrant language lateralization in down syndrome. Ann. Neurol. S114.

Shiohama, T., Levman, J., Baumer, N., and Takahashi, E. (2019). Structural magnetic resonance imaging-based brain morphology study in infants and toddlers with down syndrome: the effect of comorbidities. Pediatr. Neurol. 100, 67-73. doi: 10.1016/j.pediatrneurol.2019.03.015

Snyder, A. Z., and Raichle, M. E. (2012). A brief history of the resting state: the Washington University perspective. Neuroimage 62, 902-910. doi: 10.1016/j.neuroimage.2012.01.044

Stevenson, R. E., Schwartz, C. E., and Rogers, R. C. (2012). Atlas of Xlinked Intellectual Disability Syndromes. Oxford: Oxford University Press. doi: 10.1093/med/9780199811793.001.0001

Takashima, S., Becker, L. E., Armstrong, D. L., and Chan, F. (1981). Abnormal neuronal development in the visual cortex of the human fetus and infant with 
down's syndrome. A quantitative and qualitative Golgi study. Brain Res. 225, 1-21. doi: 10.1016/0006-8993(81)90314-0

Thornton-Wells, T. A., Cannistraci, C. J., Anderson, A. W., Kim, C. Y., Eapen, M., Gore, J. C., et al. (2010). Auditory attraction: activation of visual cortex by music and sound in Williams syndrome. Am. J. Intellect. Dev. Disabil. 115, 172-189. doi: 10.1352/1944-7588-115.172

Van Dijk, K. R. A., Hedden, T., Venkataraman, A., Evans, K. C., Lazar, S. W., and Buckner, R. L. (2010). Intrinsic functional connectivity as a tool for human connectomics: theory, properties, and optimization. J. Neurophysiol. 103, 297-321. doi: 10.1152/jn.00783.2009

*Vega, J. N., Hohman, T. J., Pryweller, J. R., Dykens, E. M., and ThorntonWells, T. A. (2015). Resting-state functional connectivity in individuals with Down syndrome and Williams syndrome compared with typically developing controls. Brain Connect. 5, 461-475. doi: 10.1089/brain. 2014.0266

Venuti, P., Caria, A., Esposito, G., De Pisapia, N., Bornstein, M. H., and de Falco, S. (2012). Differential brain responses to cries of infants with autistic disorder and typical development: an fMRI study. Res. Dev. Disabil. 33, 2255-2264. doi: 10.1016/j.ridd.2012.06.011

Virji-Babul, N., Moiseev, A., Sun, W., Feng, T., Moiseeva, N., Watt, K. J., et al. (2013). Neural correlates of music recognition in Down syndrome. Brain Cognition 81, 256-262. doi: 10.1016/j.bandc.2012.11.007

*Wan, Y. T., Chiang, C. S., Chen, S. C., and Wuang, Y. P. (2017). The effectiveness of the computerized visual perceptual training program on individuals with down syndrome: an fMRI study. Res. Dev. Disabil. 66, 1-15. doi: 10.1016/j.ridd.2017.04.015

Welvaert, M., and Rosseel, Y. (2014). A review of fMRI simulation studies. PLoS ONE 9:e101953. doi: 10.1371/journal.pone.0101953

*Wilson, L. R., Vatansever, D., Annus, T., Williams, G. B., Hong, Y. T., Fryer, T. D., et al. (2019). Differential effects of down's syndrome and Alzheimer's neuropathology on default mode connectivity. Hum. Brain Mapp. 40, 4551-4563. doi: 10.1002/hbm.24720

Wiseman, F. K., Al-Janabi, T., Hardy, J., Karmiloff-Smith, A., Nizetic, D., Tybulewicz, V. L., et al. (2015). A genetic cause of Alzheimer disease: mechanistic insights from down syndrome. Nat. Rev. Neurosci. 16:564. doi: 10.1038/nrn3983

Conflict of Interest: The authors declare that the research was conducted in the absence of any commercial or financial relationships that could be construed as a potential conflict of interest.

Copyright (C) 2020 Carbó-Carreté, Cañete-Massé, Peró-Cebollero and GuàrdiaOlmos. This is an open-access article distributed under the terms of the Creative Commons Attribution License (CC BY). The use, distribution or reproduction in other forums is permitted, provided the original author(s) and the copyright owner(s) are credited and that the original publication in this journal is cited, in accordance with accepted academic practice. No use, distribution or reproduction is permitted which does not comply with these terms. 